\title{
Vegetable, fruit and nitrate intake in relation to the risk of Barrett's oesophagus in a large Dutch cohort
}

\author{
András. P. Keszei ${ }^{1 *}$, Leo J. Schouten ${ }^{1}$, Ann L. C. Driessen ${ }^{2}$, Clément J. R. Huysentruyt ${ }^{2}$, \\ Yolande C. A. Keulemans ${ }^{3}$, R. Alexandra Goldbohm ${ }^{4}$ and Piet A. van den Brandt ${ }^{1}$ \\ ${ }^{1}$ Department of Epidemiology, School for Oncology and Developmental Biology (Grow), Maastricht University, PO Box 616, \\ 6200 MD Maastricht, The Netherlands \\ ${ }^{2}$ Department of Pathology, School for Oncology and Developmental Biology (Grow), Maastricht University, PO Box 5800, \\ 6202 AZ Maastricht, The Netherlands \\ ${ }^{3}$ Department of Gastroenterology, Maastricht University Medical Centre, P. Debyelaan 25, 6229 HX Maastricht, \\ The Netherlands \\ ${ }^{4}$ TNO, PO Box 2215, 2301 CE Leiden, The Netherlands \\ (Submitted 23 May 2013 - Final revision received 10 September 2013 - Accepted 29 October 2013 - First published online 5 December 2013)
}

\begin{abstract}
There are few epidemiological data on the dietary risk factors of Barrett's oesophagus, a precursor of oesophageal adenocarcinoma. The present study investigated the association between vegetable, fruit and nitrate intake and Barrett's oesophagus risk in a large prospective cohort. The Netherlands Cohort Study recruited 120852 individuals aged 55-69 years in 1986. Vegetable and fruit intake was assessed using a 150-item FFQ, and nitrate intake from dietary sources and drinking water was determined. After 16.3 years of follow-up, 433 cases (241 men and 192 women) of Barrett's oesophagus with specialised intestinal metaplasia and 3717 subcohort members were analysed in a case-cohort design using Cox proportional hazards models while adjusting for potential confounders. Men exhibited a lower risk of Barrett's oesophagus in the highest $v$. the lowest quintile of total (multivariable-adjusted hazard ratio (HR): $0.66,95 \%$ CI $0.43,1 \cdot 01$ ), raw (HR 0.63, 95\% CI 0.40, 0.99), raw leafy (HR 0.55, $95 \%$ CI 0.36, 0.86) and Brassica (HR 0.64, 95\% CI 0.41, 1.00) vegetable intake. No association was found for other vegetable groups and fruits. No significant associations were found between vegetable and fruit intake and Barrett's oesophagus risk among women. Total nitrate intake was inversely associated with Barrett's disease risk in men (HR $0.50,95 \%$ CI $0 \cdot 25,0.99)$ and positively associated with it in women (HR 3.77, $95 \%$ CI 1.68, 8.45) $(P$ for interaction $=0 \cdot 04)$. These results suggest that vegetable intake may contribute to the prevention of Barrett's oesophagus. The possible differential effect in men and women should be evaluated further.
\end{abstract}

Key words: Barrett's oesophagus: Vegetables: Fruits: Nitrate: Cohort studies

The incidence of oesophageal adenocarcinoma has risen in recent decades in several developed countries of the world $^{(1-5)}$. Barrett's oesophagus, a premalignant metaplastic condition of the distal oesophagus, is a precursor of oesophageal adenocarcinoma ${ }^{(6)}$. Chronic gastro-oesophageal reflux, male sex, Caucasian ethnicity, age, obesity and cigarette smoking are risk factors for the development of Barrett's oesophagus $^{(7)}$. The majority of these risk factors are difficult or impossible to modify. The identification of modifiable environmental risk factors of Barrett's disease, including dietary factors, may help in the formulation of prevention strategies for oesophageal adenocarcinoma.

Few studies have focused on dietary factors in relation to Barrett's oesophagus risk $^{(8)}$, and they have suggested an inverse association with vegetable and fruit consumption. However, no prospective cohort study has investigated this relationship. Case-control studies are prone to bias due to the possibility that individuals with preclinical symptoms may voluntarily, or on a physician's advice, change their diet.

Green vegetables are a major source of dietary nitrate intake. Nitrate may have several beneficial health effects mediated through reactive $\mathrm{N}$ intermediates, including antibacterial effects and effects on gastric mucosal integrity ${ }^{(9)}$. Nitrate content may explain part of the potentially protective effect of vegetables in various health conditions. On the other hand, nitrate is also involved in the endogenous formation of $\mathrm{N}$-nitroso compounds through reduction by anaerobic bacteria to nitrite and subsequent formation of nitrosating agents ${ }^{(10)}$.

Abbreviation: HR, hazard ratio.

*Corresponding author: Dr A. P. Keszei, fax +31 43388 4128, email andras.keszei@maastrichtuniversity.nl 
The $N$-nitrosation process can be inhibited by antioxidants such as vitamin C. Epidemiological studies have not shown an association of nitrate intake $\mathrm{i}^{(11,12)}$, or nitrate from plant sources $^{(13)}$, with oesophageal adenocarcinoma. No study has evaluated the association between nitrate intake and Barrett's oesophagus risk.

The aim of the present study was to investigate the association between vegetable and fruit consumption, as well as nitrate intake, and Barrett's oesophagus risk in a large prospective cohort of Dutch men and women.

\section{Subjects and methods}

\section{Population and follow-up}

The Netherlands Cohort Study was started in September 1986. At baseline, 58279 men and 62573 women aged 55-69 years were recruited, and a self-administered questionnaire was completed by the study participants ${ }^{(14)}$. Ethical approval to conduct the study was obtained from the institutional review boards of the University Hospital Maastricht and TNO Nutrition and Food Research. The cohort was followed for $16 \cdot 3$ years until 31 December 2002 for incident Barrett's disease cases by computerised record linkage to the nationwide network and registry of histo- and cytopathology in The Netherlands (PALGA) ${ }^{(15)}$. After manual check for false-positive linkages, excerpts of all pathology records of cases were reviewed independently by a pathologist (A. L. C. D.) and a pathologist in training (C. J. R. H.) who were blinded to exposure. Of 974 cases, 106 were excluded due to uncertain diagnosis, seventy-six due to prevalent cancer or Barrett's disease at baseline, fifty-eight due to oesophageal or gastric cancer diagnosed before or within half a year of Barrett's disease diagnosis, and 148 cases because of incomplete or inconsistent dietary data or missing data on confounders. Histology was not completely specified in the excerpts available from the central PALGA database for 250 cases. Full pathology reports of these cases were retrieved from the local pathology laboratories and were reviewed by the pathologists to identify the type of metaplasia. A total of 603 cases were available for analysis, of which 433 cases had specialised intestinal metaplasia. A case-cohort design was employed and hence a random subcohort ( $n$ 5000) was selected at baseline. Due to prevalent cancer or Barrett's disease at baseline and due to inconsistent or missing data, 230 and 1053 subcohort members were excluded from further analysis, respectively.

\section{Assessment of determinants}

Vegetable and fruit intake. Food and beverage intake during the year preceding the Netherlands Cohort Study baseline was assessed using a 150-item semi-quantitative FFQ. The participants were queried about vegetable consumption frequency in summer and that in winter separately ${ }^{(16)}$. The participants could choose one of six categories ranging from 'never or less than once per month' to 'three to seven times per week'. The subjects were asked about usual serving sizes only for string beans and cooked endive; the mean of these values served as an indicator to derive the serving sizes of all the cooked vegetables using a vegetable-specific factor calculated based on the results of a pilot study, which showed an intra-individual correlation between serving sizes of different vegetables. Vegetables that were eaten regularly, but were not specifically queried about in the questionnaire, could be entered in an open-ended question along with consumption frequency and amount consumed on each occasion. For most of the vegetables, the questionnaire explicitly specified whether the vegetable was eaten raw or cooked.

The participants were queried about fruit consumption frequency and amount of fruits consumed using categories ranging from 'never or less than once per month' to 'six or seven times per week'.

Data were key-entered and processed in a standardised manner, blinded to case/subcohort status. Mean daily intakes were calculated from frequencies and serving sizes ${ }^{(16)}$

The FFQ was validated against a $9 \mathrm{~d}$ diet record ${ }^{(17)}$. The Spearman correlation coefficients were 0.38 for total vegetable consumption and 0.60 for total fruit consumption. The FFQ appeared to slightly overestimate vegetable consumption, on average, while underestimating fruit consumption when compared with the diet records.

Nitrate intake from diet. Food composition values for nitrate were derived from the databank on contaminants in food from the State Institute for Quality Control of Agricultural Products (RIKILT). Estimations were based on the mean nitrate contents between 1985 and 1989. Distinction between summer and winter was made while calculating nitrate intake from some vegetables (i.e. endive (raw/cooked) and lettuce), and information on nitrate losses during preparation (washing, cutting or cooking) was considered. For several vegetables, experimental data were available regarding nitrate losses during preparation ${ }^{(18,19)}$. Nitrate loss percentages used to construct the nitrate table were 16, 31, 42, 20 and $49 \%$ for endive, spinach, chicory, cabbage and potatoes, respectively. For other vegetables consumed after cooking, nitrate losses were estimated to be $40 \%$. For lettuce, a $20 \%$ loss was estimated.

Nitrate intake from drinking water. Information on nitrate content in drinking water from all the pumping stations in The Netherlands in 1986 (Vereniging van Exploitanten van Waterleiding bedrijven in Nederland (VEWIN) 1989) was used to determine the nitrate concentration in drinking water for each home address by postal code. To calculate nitrate intake from water, we used information from the questionnaire about the amount of water, coffee, tea and soup consumed. Total nitrate intake was calculated by summing dietary nitrate intake and nitrate intake from water. In the subcohort, the median proportion of dietary nitrate to total nitrate was $99 \%$, and the median proportion of intake from vegetables was $90 \%$. The major source of nitrate intake among vegetables was leafy vegetables in line with studies of nitrate intake in the Dutch population $^{(20)}$.

Vitamin C intake. Daily vitamin C intake was calculated from the FFQ data using the Dutch food composition table. 
Assessment of potential confounders. Information on education (primary school, lower vocational, high school or higher vocational/university), cigarette smoking status (never-smoker, ex-smoker or current smoker), smoking history (number of cigarettes smoked and duration of smoking), total energy intake $(\mathrm{kJ} / \mathrm{d})$, BMI $\left(\mathrm{kg} / \mathrm{m}^{2}\right)$, non-occupational physical activity $(<30,30-60,60-90$ or $>90 \mathrm{~min} / \mathrm{d})$, alcohol intake $(\mathrm{g} / \mathrm{d})$, and long-term (more than half year) use of nonsteroidal anti-inflammatory drugs and lower oesophageal sphincter-relaxing medications (nitroglycerins, aminophyllines, $\beta$-blockers, anticholinergics, nifedipine and benzodiazepines) was obtained from the baseline questionnaire.

\section{Statistical analyses}

The characteristics of cases and subcohort members are described using percentages, means, standard deviations, medians and interquartile ranges. Incidence rate ratios and 95\% CI for Barrett's oesophagus were estimated using Cox proportional hazards models comparing quintiles of intakes and using continuous variables. Analyses were carried out using Prentice's method of weighting for case-cohort designs ${ }^{(21)}$. Men and women were analysed separately. Standard errors were calculated using a robust variance estimator ${ }^{(22)}$. Results were similar while using time on study and age as the time scale; hence, only results with the former are presented. Tests

Table 1. Characteristics of Barrett's disease cases and subcohort members in the Netherlands Cohort Study on diet and cancer, 1986-2002 (Median values and interquartile ranges (IQR))

\begin{tabular}{|c|c|c|c|c|c|c|c|c|}
\hline \multirow[b]{3}{*}{ Characteristics } & \multicolumn{4}{|c|}{ Men } & \multicolumn{4}{|c|}{ Women } \\
\hline & \multicolumn{2}{|c|}{$\begin{array}{l}\text { Subcohort } \\
(n 1833)\end{array}$} & \multicolumn{2}{|c|}{$\begin{array}{l}\text { Cases } \\
(n 241)\end{array}$} & \multicolumn{2}{|c|}{$\begin{array}{l}\text { Subcohort } \\
(n 1884)\end{array}$} & \multicolumn{2}{|c|}{$\begin{array}{l}\text { Cases } \\
(n 192)\end{array}$} \\
\hline & Median & IQR & Median & IQR & Median & IQR & Median & IQR \\
\hline \multicolumn{9}{|l|}{ Age (years) } \\
\hline Mean & \multicolumn{2}{|c|}{$61 \cdot 2$} & \multicolumn{2}{|r|}{$61 \cdot 1$} & \multicolumn{2}{|r|}{61.4} & \multicolumn{2}{|r|}{61.4} \\
\hline SD & \multicolumn{2}{|r|}{$4 \cdot 2$} & \multicolumn{2}{|r|}{$4 \cdot 1$} & \multicolumn{2}{|r|}{4.2} & \multicolumn{2}{|r|}{4.1} \\
\hline \multicolumn{9}{|l|}{$\mathrm{BMI}\left(\mathrm{kg} / \mathrm{m}^{2}\right)$} \\
\hline Mean & \multirow{2}{*}{\multicolumn{2}{|c|}{$\begin{array}{c}24.9 \\
2.6\end{array}$}} & \multicolumn{2}{|r|}{$25 \cdot 0$} & \multicolumn{2}{|r|}{$25 \cdot 0$} & \multicolumn{2}{|r|}{$26 \cdot 1$} \\
\hline SD & & & \multicolumn{2}{|r|}{$2 \cdot 4$} & & 3.5 & & $3 \cdot 0$ \\
\hline Cigarette smoking (\%) & \multicolumn{2}{|r|}{$2 \cdot 6$} & & & & & & \\
\hline Never-smoker & & 14 & & 12 & & 59 & & 61 \\
\hline Former smoker & & 53 & & 61 & & 20 & & 26 \\
\hline Current smoker & & 33 & & 27 & & 21 & & 14 \\
\hline Level of education (\%) & & & & & & & & \\
\hline Primary school & & 23 & & 24 & & 32 & & 35 \\
\hline Lower vocational & & 20 & & 17 & & 23 & & 24 \\
\hline High school & & 37 & & 34 & & 36 & & 35 \\
\hline Higher vocational/university & & 20 & & 24 & & 10 & & 6 \\
\hline Non-occupational physical activity (\%) & & & & & & & & \\
\hline$<30 \mathrm{~min} / \mathrm{d}$ & & 17 & & 18 & & 23 & & 31 \\
\hline $30-<60 \mathrm{~min} / \mathrm{d}$ & & 31 & & 27 & & 32 & & 30 \\
\hline $60-<90 \mathrm{~min} / \mathrm{d}$ & & 20 & & 23 & & 23 & & 21 \\
\hline$\geq 90 \mathrm{~min} / \mathrm{d}$ & & 32 & & 32 & & 22 & & 18 \\
\hline $\begin{array}{l}\text { Family history of oesophageal or } \\
\text { gastric cancer (\%) }\end{array}$ & & 8 & & 7 & & 7 & & 6 \\
\hline Use of non-steroidal anti-inflammatory drugs (\%) & & 5 & & 8 & & 8 & & 11 \\
\hline $\begin{array}{l}\text { Use of lower oesophageal } \\
\text { sphincter-relaxing medication (\%) }\end{array}$ & & 15 & & 18 & & 14 & & 17 \\
\hline Daily intake in subcohort ${ }^{\star} \dagger$ & & & & & & & & \\
\hline Alcohol $(\mathrm{g} / \mathrm{d})$ & 9.7 & $2 \cdot 2-23 \cdot 1$ & $9 \cdot 6$ & $2 \cdot 2-22 \cdot 7$ & 1.7 & $0-8.0$ & 1.1 & $0-3.7$ \\
\hline Energy $(\mathrm{kJ} / \mathrm{d})$ & & & & & & & & \\
\hline Mean & & 9065 & & 8931 & & 7045 & & 6759 \\
\hline SD & & 2087 & & 2092 & & 1628 & & 1643 \\
\hline Fruits $(g / d)$ & 136 & $76-210$ & 134 & $77-218$ & 177 & $113-257$ & 177 & $116-268$ \\
\hline Vegetables $(\mathrm{g} / \mathrm{d})$ & 177 & $136-227$ & 178 & $122-220$ & 182 & $141-231$ & 181 & $140-236$ \\
\hline Raw vegetables (g/d) & $30 \cdot 7$ & $16 \cdot 6-49 \cdot 5$ & $27 \cdot 3$ & $14 \cdot 2-45 \cdot 4$ & 36.7 & $21 \cdot 6-56 \cdot 8$ & 32.8 & $17 \cdot 8-53 \cdot 5$ \\
\hline Cooked vegetables $(\mathrm{g} / \mathrm{d})$ & $143 \cdot 1$ & $106.5-184.5$ & $138 \cdot 0$ & $101 \cdot 0-177 \cdot 8$ & 142.9 & $109 \cdot 0-183 \cdot 3$ & $147 \cdot 8$ & $111 \cdot 3-174 \cdot 2$ \\
\hline Raw leafy vegetables $(\mathrm{g} / \mathrm{d})$ & $7 \cdot 1$ & $3.6-13 \cdot 9$ & 6.4 & $3.6-11.5$ & $7 \cdot 2$ & $3 \cdot 6-14 \cdot 2$ & $7 \cdot 1$ & $3 \cdot 6-14 \cdot 2$ \\
\hline Cooked leafy vegetables $(\mathrm{g} / \mathrm{d})$ & 18.7 & $10 \cdot 7-29 \cdot 2$ & $17 \cdot 2$ & $9 \cdot 3-27 \cdot 3$ & $19 \cdot 0$ & $10 \cdot 0-28 \cdot 7$ & $20 \cdot 4$ & $13 \cdot 7-29 \cdot 8$ \\
\hline Allium vegetables $(\mathrm{g} / \mathrm{d})$ & 22.5 & $11 \cdot 0-40 \cdot 2$ & 21.9 & $11 \cdot 0-40 \cdot 2$ & $25 \cdot 3$ & $11 \cdot 0-42 \cdot 3$ & $22 \cdot 3$ & $12 \cdot 4-41 \cdot 7$ \\
\hline Legumes $(\mathrm{g} / \mathrm{d})$ & 29.9 & $19 \cdot 4-44 \cdot 3$ & 28.5 & $16 \cdot 6-43 \cdot 5$ & $26 \cdot 7$ & $16 \cdot 8-39 \cdot 7$ & 25.5 & $14 \cdot 6-36 \cdot 5$ \\
\hline Brassica vegetables $(\mathrm{g} / \mathrm{d})$ & $29 \cdot 0$ & $18 \cdot 8-42 \cdot 8$ & 27.5 & $16 \cdot 9-40 \cdot 6$ & 27.9 & $17.9-40.5$ & 28.8 & $14 \cdot 8-40 \cdot 5$ \\
\hline Citrus fruits (g/d) & $42 \cdot 0$ & $11 \cdot 4-94.6$ & $46 \cdot 8$ & $11 \cdot 2-90 \cdot 1$ & $76 \cdot 6$ & $29 \cdot 7-124 \cdot 4$ & $69 \cdot 1$ & $30.5-118.5$ \\
\hline Nitrate from food $(\mathrm{mg} / \mathrm{d})$ & 98.8 & $75 \cdot 7-125 \cdot 2$ & $95 \cdot 1$ & $70 \cdot 8-114 \cdot 8$ & $96 \cdot 6$ & $73 \cdot 1-123 \cdot 9$ & $96 \cdot 2$ & $77 \cdot 9-121 \cdot 1$ \\
\hline Nitrate from water $(\mathrm{mg} / \mathrm{d})$ & $1 \cdot 2$ & $0.4-2.2$ & 1.4 & $0.6-2.6$ & 1.2 & $0.4-2.3$ & 1.3 & $0.5-2.3$ \\
\hline Total nitrate $(\mathrm{mg} / \mathrm{d})$ & $100 \cdot 4$ & $76 \cdot 9-126 \cdot 9$ & $96 \cdot 7$ & $72 \cdot 9-117 \cdot 6$ & 97.8 & $74 \cdot 8-125 \cdot 7$ & $97 \cdot 1$ & $78 \cdot 9-123 \cdot 6$ \\
\hline
\end{tabular}

*Vegetables included string/French beans, cauliflower, lettuce, carrots (raw/cooked), endive (raw/cooked), Brussels sprouts, sauerkraut, tomatoes, onions, spinach, beetroot, kale, cabbage, leek, mushrooms, broad beans, sweet peppers, rhubarb and gherkins.

†Fruits included apples, pears, strawberries, oranges and fresh orange juice, grapes, mandarins, bananas, grapefruits and grapefruit juice, raisins, and other dried fruits. 
Table 2. Hazard ratios (HR) for Barrett's oesophagus with specialised intestinal metaplasia in men by vegetable and fruit intake in the Netherlands Cohort Study on diet and cancer, 1986-2002

(Hazard ratios and $95 \%$ confidence intervals)

\begin{tabular}{|c|c|c|c|c|c|c|c|}
\hline & \multirow[b]{2}{*}{ Cases $(n)$} & \multicolumn{2}{|c|}{ Subcohort } & \multirow[b]{2}{*}{$\mathrm{HR}^{*}$} & \multirow[b]{2}{*}{$95 \% \mathrm{Cl}$} & \multirow[b]{2}{*}{$\mathrm{HR} \dagger$} & \multirow[b]{2}{*}{$95 \% \mathrm{Cl}$} \\
\hline & & Person-years & Median intake $(\mathrm{g} / \mathrm{d})$ & & & & \\
\hline \multicolumn{8}{|l|}{ Total vegetable intake } \\
\hline Q1 & 66 & 4875 & 103.5 & 1 & & 1 & \\
\hline Q2 & 39 & 5279 & $144 \cdot 1$ & 0.53 & $0.35,0.82$ & 0.54 & $0.35,0.83$ \\
\hline Q3 & 59 & 5167 & $178 \cdot 2$ & 0.83 & $0.57,1.22$ & 0.84 & $0.57,1.24$ \\
\hline Q4 & 34 & 5163 & $216 \cdot 9$ & 0.48 & $0.31,0.74$ & 0.47 & $0.30,0.74$ \\
\hline Q5 & 43 & 4715 & 287.4 & 0.66 & $0.43,1.00$ & 0.66 & $0.43,1.01$ \\
\hline$P$ for trend & & & & & 0.07 & & 0.08 \\
\hline Continuous ( $25 \mathrm{~g} / \mathrm{d}$ increment) & & & & 0.96 & $0.91,1.01$ & 0.96 & $0.91,1.01$ \\
\hline \multicolumn{8}{|l|}{ Raw vegetable intake } \\
\hline Q1 & 57 & 4851 & $6 \cdot 9$ & 1 & & 1 & \\
\hline Q2 & 55 & 5003 & $19 \cdot 2$ & 0.92 & $0.62,1.38$ & 0.94 & $0.62,1.42$ \\
\hline Q3 & 51 & 5003 & $30 \cdot 6$ & 0.86 & $0.57,1.29$ & 0.85 & $0.55,1.29$ \\
\hline Q4 & 40 & 5346 & $45 \cdot 5$ & 0.62 & $0.40,0.96$ & 0.62 & $0.40,0.98$ \\
\hline Q5 & 38 & 4996 & 73.1 & 0.63 & $0.41,0.98$ & 0.63 & $0.40,0.99$ \\
\hline$P$ for trend & & & & & 0.01 & & 0.02 \\
\hline Continuous ( $25 \mathrm{~g} / \mathrm{d}$ increment) & & & & 0.87 & $0.76,1.00$ & 0.87 & $0.75,1.01$ \\
\hline \multicolumn{8}{|l|}{ Cooked vegetable intake } \\
\hline Q1 & 57 & 4965 & $79 \cdot 3$ & 1 & & 1 & \\
\hline Q2 & 46 & 5204 & 114.9 & 0.75 & $0.50,1.15$ & 0.76 & $0.50,1.17$ \\
\hline Q3 & 50 & 5255 & $143 \cdot 8$ & 0.82 & $0.54,1.23$ & 0.81 & $0.53,1.22$ \\
\hline Q4 & 46 & 5130 & 176.9 & 0.77 & $0.50,1.17$ & 0.75 & $0.49,1.16$ \\
\hline Q5 & 42 & 4645 & $236 \cdot 3$ & 0.77 & $0.50,1 \cdot 19$ & 0.78 & $0.50,1.21$ \\
\hline$P$ for trend & & & & & 0.33 & & 0.34 \\
\hline Continuous ( $25 \mathrm{~g} / \mathrm{d}$ increment) & & & & 0.97 & $0.91,1.03$ & 0.97 & $0.91,1.03$ \\
\hline \multicolumn{8}{|l|}{ Raw leafy vegetable intake } \\
\hline Q1 & 76 & 6232 & $2 \cdot 0$ & 1 & & 1 & \\
\hline Q2 & 42 & 3578 & 4.4 & 0.95 & $0.63,1.44$ & 0.96 & $0.63,1.45$ \\
\hline Q3 & 46 & 5168 & $7 \cdot 1$ & 0.72 & $0.48,1.06$ & 0.71 & $0.47,1.05$ \\
\hline Q4 & 41 & 5078 & 11.8 & 0.66 & $0.44,0.99$ & 0.66 & $0.43,1.00$ \\
\hline Q5 & 36 & 5142 & $21 \cdot 6$ & 0.56 & $0.37,0.85$ & 0.55 & $0.36,0.86$ \\
\hline$P$ for trend & & & & & 0.004 & & 0.005 \\
\hline Continuous ( $25 \mathrm{~g} / \mathrm{d}$ increment) & & & & 0.55 & $0.35,0.88$ & 0.55 & $0.34,0.89$ \\
\hline \multicolumn{8}{|l|}{ Cooked leafy vegetable intake } \\
\hline Q1 & 56 & 4982 & $4 \cdot 3$ & 1 & & 1 & \\
\hline Q2 & 50 & 5245 & $12 \cdot 3$ & 0.84 & $0.55,1.26$ & 0.82 & $0.53,1.26$ \\
\hline Q3 & 47 & 5008 & $18 \cdot 8$ & 0.84 & $0.55,1.28$ & 0.79 & $0.51,1.22$ \\
\hline Q4 & 42 & 5185 & $27 \cdot 1$ & 0.71 & $0.47,1.10$ & 0.69 & $0.45,1.07$ \\
\hline Q5 & 46 & 4779 & $41 \cdot 8$ & 0.85 & $0.56,1.30$ & $0 \cdot 84$ & $0.55,1 \cdot 29$ \\
\hline$P$ for trend & & & & & 0.42 & & 0.39 \\
\hline Continuous ( $25 \mathrm{~g} / \mathrm{d}$ increment) & & & & 0.85 & $0.68,1.07$ & 0.85 & $0.67,1.07$ \\
\hline \multicolumn{8}{|l|}{ Allium vegetable intake } \\
\hline Q1 & 73 & 7030 & $5 \cdot 3$ & 1 & & 1 & \\
\hline Q2 & 28 & 3126 & $15 \cdot 5$ & 0.86 & $0.54,1.37$ & 0.85 & $0.53,1.36$ \\
\hline Q3 & 51 & 5301 & 23.7 & 0.92 & $0.62,1.34$ & 0.90 & $0.61,1.33$ \\
\hline Q4 & 46 & 5070 & $36 \cdot 8$ & 0.86 & $0.58,1.28$ & 0.85 & $0.56,1.28$ \\
\hline Q5 & 43 & 4672 & $61 \cdot 2$ & 0.87 & $0.58,1.31$ & 0.87 & $0.58,1.33$ \\
\hline$P$ for trend & & & & & 0.53 & & 0.55 \\
\hline Continuous ( $25 \mathrm{~g} / \mathrm{d}$ increment) & & & & 0.95 & $0.82,1.11$ & 0.95 & $0.81,1 \cdot 12$ \\
\hline Legume intake & & & & & & & \\
\hline Q1 & 66 & 5080 & $11 \cdot 6$ & 1 & & 1 & \\
\hline Q2 & 40 & 5101 & 21.5 & 0.61 & $0.40,0.94$ & 0.63 & $0.41,0.96$ \\
\hline Q3 & 50 & 5141 & 29.9 & 0.75 & $0.51,1.12$ & 0.77 & $0.51,1.16$ \\
\hline Q4 & 42 & 5167 & 40.9 & 0.64 & $0.42,0.97$ & 0.63 & $0.41,0.96$ \\
\hline Q5 & 43 & 4710 & 63.2 & 0.72 & $0.47,1.08$ & 0.73 & $0.48,1.12$ \\
\hline$P$ for trend & & & & & 0.22 & & 0.24 \\
\hline Continuous ( $25 \mathrm{~g} / \mathrm{d}$ increment) & & & & 0.89 & $0.76,1.04$ & 0.89 & $0.76,1.05$ \\
\hline Brassica vegetable intake & & & & & & & \\
\hline Q1 & 57 & 4954 & $10 \cdot 7$ & 1 & & 1 & \\
\hline Q2 & 52 & 5266 & $21 \cdot 0$ & 0.84 & $0.56,1.27$ & 0.87 & $0.57,1.32$ \\
\hline Q3 & 44 & 5062 & $29 \cdot 3$ & 0.74 & $0.49,1.13$ & 0.74 & $0.48,1.13$ \\
\hline Q4 & 52 & 5161 & 39.8 & 0.86 & $0.57,1.29$ & 0.86 & $0.57,1.30$ \\
\hline Q5 & 36 & 4756 & $59 \cdot 0$ & 0.65 & $0.41,1.01$ & 0.64 & $0.41,1.00$ \\
\hline$P$ for trend & & & & & 0.09 & & 0.08 \\
\hline Continuous ( $25 \mathrm{~g} / \mathrm{d}$ increment) & & & & 0.88 & $0.72,1.06$ & 0.88 & $0.72,1.07$ \\
\hline
\end{tabular}




\begin{tabular}{|c|c|c|c|c|c|c|c|}
\hline & \multirow[b]{2}{*}{ Cases $(n)$} & \multicolumn{2}{|c|}{ Subcohort } & \multirow[b]{2}{*}{$\mathrm{HR}^{\star}$} & \multirow[b]{2}{*}{$95 \% \mathrm{Cl}$} & \multirow[b]{2}{*}{$\mathrm{HR} \dagger$} & \multirow[b]{2}{*}{$95 \% \mathrm{Cl}$} \\
\hline & & Person-years & Median intake $(\mathrm{g} / \mathrm{d})$ & & & & \\
\hline \multicolumn{8}{|l|}{ Fruit intake } \\
\hline Q1 & 49 & 4653 & $31 \cdot 2$ & 1 & & 1 & \\
\hline Q2 & 53 & 5051 & 88.4 & 0.97 & $0.64,1.47$ & 0.93 & $0.61,1.42$ \\
\hline Q3 & 45 & 5062 & $134 \cdot 6$ & 0.81 & $0.53,1.25$ & 0.78 & $0.50,1.22$ \\
\hline Q4 & 36 & 5169 & $187 \cdot 2$ & 0.64 & $0.41,1.02$ & 0.64 & $0.40,1.03$ \\
\hline Q5 & 58 & 5264 & $292 \cdot 8$ & 1.00 & $0.66,1.51$ & 1.00 & $0.65,1.53$ \\
\hline$P$ for trend & & & & & 0.76 & & 0.87 \\
\hline Continuous ( $25 \mathrm{~g} / \mathrm{d}$ increment) & & & & 0.99 & $0.96,1.02$ & 0.99 & $0.96,1.02$ \\
\hline \multicolumn{8}{|l|}{ Citrus fruit intake } \\
\hline Q1 & 47 & 4877 & 0 & 1 & & 1 & \\
\hline Q2 & 47 & 5115 & $15 \cdot 7$ & 0.94 & $0.61,1.46$ & 0.96 & $0.61,1.50$ \\
\hline Q3 & 45 & 4951 & $41 \cdot 0$ & 0.92 & $0.59,1.43$ & 0.91 & $0.58,1.43$ \\
\hline Q4 & 61 & 5098 & $83 \cdot 3$ & 1.22 & $0.81,1 \cdot 84$ & 1.22 & $0.79,1.88$ \\
\hline Q5 & 41 & 5157 & $166 \cdot 7$ & 0.80 & $0.51,1 \cdot 26$ & 0.81 & $0.51,1.28$ \\
\hline$P$ for trend & & & & & 0.53 & & 0.54 \\
\hline Continuous ( $25 \mathrm{~g} / \mathrm{d}$ increment) & & & & 0.99 & $0.94,1.04$ & 0.99 & $0.94,1.04$ \\
\hline
\end{tabular}

Q, quintile.

*Adjusted for age (years); calculated using Cox proportional hazards model.

†Adjusted for age (years), smoking status (current $v$. non-current smoker), duration of cigarette smoking (years), number of cigarettes smoked per d, total energy intake (kJ/d), BMI (quintiles), alcohol intake ( $\mathrm{g} / \mathrm{d})$, levels of education (four categories), non-occupational physical activity (four categories) and use of lower oesophageal sphincter-relaxing medications (yes/no); calculated using Cox proportional hazards model.

of linear trend in the incidence rate ratios were carried out by fitting models with the median values of each exposure quintile as a continuous variable. The proportional hazards assumption was assessed using the scaled Schoenfeld residuals $^{(23)}$ and by introducing time-covariate interactions into the models and examining estimates and testing their significance using the Wald test. Substantial deviation from the assumption was not detected for any of the exposure variables. Effect modification by sex and vitamin $\mathrm{C}$ intake was tested using cross-product terms. All the models were adjusted for age, and multivariable models were additionally adjusted for smoking status (current: yes/no), number of cigarettes smoked per $\mathrm{d}$, duration of smoking (years), total energy intake $(\mathrm{kJ} / \mathrm{d})$, alcohol intake (g/d), BMI categories (quintiles), levels of education (four categories), non-occupational physical activity (four categories) and long-term use of lower oesophageal sphincter-relaxing medications (yes/no). Models for nitrate intake were also adjusted for vegetable intake $(\mathrm{g} / \mathrm{d})$. Primary analysis was carried out using only cases with intestinal metaplasia ( $n$ 433). Long-term use of reflux medications and vitamin C supplement use were also considered in multivariable models, but were not included in the final reported models as they had only minor effects on the estimates. Additional analyses were carried out restricted to individuals who reported having similar vegetable and fruit intakes 5 years before baseline. Furthermore, we carried out an analysis by excluding the first 2 years of follow-up.

Based on a method proposed by Cai \& Zeng ${ }^{(24)}$ and using the available number of cases and subcohort members, we estimated that an $80 \%$ power could be achieved to show hazard ratio (HR) of 0.55 and 0.5 between quintiles of exposure with a two-sided type 1 error of 0.05 among men and women, respectively.

\section{Results}

The baseline characteristics of 241 male and 192 female cases and 3717 subcohort members are given in Table 1. Cases were more often men, were less likely to be current smokers at baseline, and were somewhat more likely to have used lower oesophageal sphincter-relaxing medications and non-steroidal anti-inflammatory drugs. The median intake of total vegetables was $179 \mathrm{~g} / \mathrm{d}$ (interquartile range $138-229 \mathrm{~g} / \mathrm{d}$ ) in the subcohort and was higher in women than in men (Kruskal-Wallis $\left.\chi^{2}(\mathrm{df}=1): 7 \cdot 3, P=0 \cdot 007\right)$. The intakes of different groups of vegetables were comparable between cases and subcohort members (Table 1). Vegetables consumed in the largest amount in the subcohort were tomatoes, onions, string beans and cauliflower (median 19, 17, 17 and 13g/d, respectively). Median fruit consumption in the subcohort was $157 \mathrm{~g} / \mathrm{d}$ (interquartile range $94-234 \mathrm{~g} / \mathrm{d}$ ) with a higher intake in women (Kruskal-Wallis $\chi^{2}(\mathrm{df}=1)$ : $\left.153, P=0 \cdot 0001\right)$.

Total nitrate intake strongly correlated with vegetable intake in the subcohort ( $r$ 0.83) and median intake was comparable between cases and subcohort members (Table 1 ).

Among men, total vegetable intake was inversely associated with the risk of Barrett's oesophagus with intestinal metaplasia (Table 2), but a clear linear trend was not observed. A strong inverse association with a clear trend was observed with raw leafy vegetable consumption. The multivariable-adjusted model with continuous exposure variables estimated a $45 \%$ decrease in risk per $25 \mathrm{~g} / \mathrm{d}$ increase in raw leafy vegetable intake. An inverse association was also found for total raw vegetable and Brassica vegetable intake. No association was found for cooked vegetable and fruit intake.

Associations between vegetable and fruit intake and Barrett's oesophagus risk among women are summarised in Table 3. The strongest inverse association was observed for Brassica 
Table 3. Hazard ratios (HR) for Barrett's oesophagus with specialised intestinal metaplasia in women by vegetable and fruit intake in the Netherlands Cohort Study on diet and cancer, 1986-2002

(Hazard ratios and $95 \%$ confidence intervals)

\begin{tabular}{|c|c|c|c|c|c|c|c|}
\hline & \multirow[b]{2}{*}{ Cases $(n)$} & \multicolumn{2}{|c|}{ Subcohort } & \multirow[b]{2}{*}{$\mathrm{HR}^{*}$} & \multirow[b]{2}{*}{$95 \% \mathrm{Cl}$} & \multirow[b]{2}{*}{$\mathrm{HR} \dagger$} & \multirow[b]{2}{*}{$95 \% \mathrm{Cl}$} \\
\hline & & Person-years & Median intake $(\mathrm{g} / \mathrm{d})$ & & & & \\
\hline \multicolumn{8}{|l|}{ Total vegetable intake } \\
\hline Q1 & 39 & 5440 & $106 \cdot 3$ & 1 & & 1 & \\
\hline Q2 & 32 & 5799 & $148 \cdot 7$ & 0.77 & $0.47,1.25$ & 0.82 & $0.49,1.35$ \\
\hline Q3 & 47 & 5997 & $182 \cdot 8$ & 1.09 & $0.70,1.71$ & 1.21 & $0.76,1.93$ \\
\hline Q4 & 46 & 5874 & 223.6 & $1 \cdot 10$ & $0.70,1.72$ & 1.22 & $0.75,1.97$ \\
\hline Q5 & 28 & 4924 & 298.8 & 0.80 & $0.48,1.33$ & 0.98 & $0.57,1.67$ \\
\hline$P$ for trend & & & & & 0.78 & & 0.61 \\
\hline Continuous ( $25 \mathrm{~g} / \mathrm{d}$ increment) & & & & 0.98 & $0.93,1.03$ & 1.00 & $0.95,1.05$ \\
\hline \multicolumn{8}{|l|}{ Raw vegetable intake } \\
\hline Q1 & 48 & 5604 & $9 \cdot 4$ & 1 & & 1 & \\
\hline Q2 & 41 & 5413 & $24 \cdot 7$ & 0.89 & $0.57,1.38$ & 0.89 & $0.56,1.42$ \\
\hline Q3 & 37 & 5842 & $36 \cdot 5$ & 0.74 & $0.47,1.16$ & 0.84 & $0.52,1.34$ \\
\hline Q4 & 36 & 5772 & $51 \cdot 7$ & 0.73 & $0.46,1.14$ & 0.83 & $0.52,1.34$ \\
\hline Q5 & 30 & 5403 & 78.0 & 0.65 & $0.40,1.05$ & 0.77 & $0.47,1.27$ \\
\hline$P$ for trend & & & & & 0.06 & & 0.31 \\
\hline Continuous ( $25 \mathrm{~g} / \mathrm{d}$ increment) & & & & 0.90 & $0.77,1.03$ & 0.95 & $0.82,1.09$ \\
\hline \multicolumn{8}{|l|}{ Cooked vegetable intake } \\
\hline Q1 & 35 & 5426 & $80 \cdot 5$ & 1 & & 1 & \\
\hline Q2 & 36 & 5648 & $115 \cdot 9$ & 0.99 & $0.61,1.61$ & 1.04 & $0.64,1.71$ \\
\hline Q3 & 41 & 6207 & $144 \cdot 0$ & 1.01 & $0.63,1.63$ & $1 \cdot 10$ & $0.67,1.79$ \\
\hline Q4 & 47 & 5766 & $177 \cdot 7$ & 1.27 & $0.80,2.03$ & 1.36 & $0 \cdot 85,2 \cdot 19$ \\
\hline Q5 & 33 & 4987 & $234 \cdot 1$ & 1.04 & $0.63,1.70$ & $1 \cdot 18$ & $0.71,1.99$ \\
\hline$P$ for trend & & & & & 0.60 & & 0.32 \\
\hline Continuous ( $25 \mathrm{~g} / \mathrm{d}$ increment) & & & & 0.99 & $0.93,1.05$ & 1.01 & $0.95,1.07$ \\
\hline \multicolumn{8}{|l|}{ Raw leafy vegetable intake } \\
\hline Q1 & 49 & 7176 & $2 \cdot 1$ & 1 & & 1 & \\
\hline Q2 & 34 & 4225 & 4.8 & $1 \cdot 20$ & $0.75,1.90$ & 1.26 & $0.78,2.05$ \\
\hline Q3 & 39 & 5788 & $7 \cdot 6$ & 0.99 & $0.64,1.54$ & 0.99 & $0.62,1.57$ \\
\hline Q4 & 35 & 5634 & $13 \cdot 0$ & 0.92 & $0.59,1.45$ & 0.93 & $0.58,1.51$ \\
\hline Q5 & 35 & 5211 & $22 \cdot 8$ & 0.99 & $0.63,1.57$ & 1.14 & $0.70,1.84$ \\
\hline$P$ for trend & & & & & 0.71 & & 0.90 \\
\hline Continuous ( $25 \mathrm{~g} / \mathrm{d}$ increment) & & & & $1 \cdot 10$ & $0.69,1.75$ & 1.27 & $0.78,2.05$ \\
\hline \multicolumn{8}{|l|}{ Cooked leafy vegetable intake } \\
\hline Q1 & 28 & 5689 & 4.3 & 1 & & 1 & \\
\hline Q2 & 29 & 5668 & 11.9 & 1.04 & $0.61,1.78$ & 1.04 & $0.60,1.80$ \\
\hline Q3 & 51 & 5720 & $19 \cdot 2$ & 1.81 & $1.12,2.94$ & 1.95 & $1.18,3.23$ \\
\hline Q4 & 45 & 5585 & $27 \cdot 0$ & 1.64 & $1.00,2.69$ & 1.71 & $1 \cdot 02,2 \cdot 86$ \\
\hline Q5 & 39 & 5372 & $41 \cdot 8$ & 1.47 & $0.89,2.45$ & 1.53 & $0.91,2.59$ \\
\hline$P$ for trend & & & & & 0.05 & & 0.04 \\
\hline Continuous ( $25 \mathrm{~g} / \mathrm{d}$ increment) & & & & $1 \cdot 10$ & $0.92,1.32$ & $1 \cdot 12$ & $0.92,1.36$ \\
\hline \multicolumn{8}{|l|}{ Allium vegetable intake } \\
\hline Q1 & 47 & 7379 & $3 \cdot 8$ & 1 & & 1 & \\
\hline Q2 & 48 & 5205 & $18 \cdot 3$ & 1.46 & $0.95,2.23$ & 1.57 & $1.01,2.42$ \\
\hline Q3 & 32 & 5013 & $27 \cdot 6$ & 1.00 & $0.62,1.59$ & $1 \cdot 12$ & $0.68,1.82$ \\
\hline Q4 & 27 & 5061 & $40 \cdot 1$ & 0.84 & $0.52,1.38$ & 0.99 & $0.59,1.65$ \\
\hline Q5 & 38 & 5377 & $60 \cdot 3$ & 1.11 & $0.71,1.75$ & 1.27 & $0.79,2.03$ \\
\hline$P$ for trend & & & & & 0.80 & & 0.69 \\
\hline Continuous ( $25 \mathrm{~g} / \mathrm{d}$ increment) & & & & 1.01 & $0.86,1 \cdot 19$ & 1.06 & $0.90,1.25$ \\
\hline Legume intake & & & & & & & \\
\hline Q1 & 45 & 5241 & $10 \cdot 2$ & 1 & & 1 & \\
\hline Q2 & 28 & 5960 & $18 \cdot 0$ & 0.55 & $0.33,0.90$ & 0.56 & $0.34,0.93$ \\
\hline Q3 & 48 & 5638 & $26 \cdot 6$ & 1.00 & $0.65,1.54$ & 1.02 & $0.64,1.62$ \\
\hline Q4 & 41 & 5828 & $37 \cdot 0$ & 0.83 & $0.53,1.29$ & 0.83 & $0.52,1.32$ \\
\hline Q5 & 30 & 5367 & $58 \cdot 9$ & 0.66 & $0.41,1.07$ & 0.71 & $0.42,1.20$ \\
\hline$P$ for trend & & & & & 0.32 & & 0.49 \\
\hline Continuous ( $25 \mathrm{~g} / \mathrm{d}$ increment) & & & & 0.81 & $0.67,0.98$ & 0.83 & $0.67,1.01$ \\
\hline Brassica vegetable intake & & & & & & & \\
\hline Q1 & 50 & 5454 & $10 \cdot 5$ & 1 & & 1 & \\
\hline Q2 & 32 & 5784 & $19 \cdot 8$ & 0.60 & $0.38,0.96$ & 0.61 & $0.38,0.99$ \\
\hline Q3 & 32 & 5851 & $28 \cdot 2$ & 0.60 & $0.38,0.95$ & 0.60 & $0.37,0.98$ \\
\hline Q4 & 47 & 5699 & $38 \cdot 2$ & 0.91 & $0.59,1.39$ & 0.93 & $0.60,1.45$ \\
\hline Q5 & 31 & 5246 & $57 \cdot 8$ & 0.65 & $0.41,1.05$ & 0.65 & $0.40,1.07$ \\
\hline$P$ for trend & & & & & 0.37 & & 0.40 \\
\hline Continuous ( $25 \mathrm{~g} / \mathrm{d}$ increment) & & & & 0.88 & $0.72,1.08$ & 0.89 & $0.71,1.11$ \\
\hline
\end{tabular}


Table 3. Continued

\begin{tabular}{|c|c|c|c|c|c|c|c|}
\hline & \multirow[b]{2}{*}{ Cases $(n)$} & \multicolumn{2}{|c|}{ Subcohort } & \multirow[b]{2}{*}{$\mathrm{HR}^{\star}$} & \multirow[b]{2}{*}{$95 \% \mathrm{Cl}$} & \multirow[b]{2}{*}{$\mathrm{HR} \dagger$} & \multirow[b]{2}{*}{$95 \% \mathrm{Cl}$} \\
\hline & & Person-years & Median intake $(\mathrm{g} / \mathrm{d})$ & & & & \\
\hline \multicolumn{8}{|l|}{ Fruit intake } \\
\hline Q1 & 43 & 5538 & 64.5 & 1 & & 1 & \\
\hline Q2 & 36 & 5564 & 124.9 & 0.84 & $0.53,1.34$ & 0.93 & $0.58,1.52$ \\
\hline Q3 & 29 & 5680 & $176 \cdot 9$ & 0.65 & $0.40,1.07$ & 0.72 & $0.43,1.20$ \\
\hline Q4 & 41 & 5800 & $236 \cdot 8$ & 0.91 & $0.58,1.42$ & 0.91 & $0.57,1.46$ \\
\hline Q5 & 43 & 5451 & $342 \cdot 6$ & 1.01 & $0.64,1.58$ & $1 \cdot 12$ & $0 \cdot 69,1 \cdot 81$ \\
\hline$P$ for trend & & & & & 0.74 & & 0.58 \\
\hline Continuous ( $25 \mathrm{~g} / \mathrm{d}$ increment) & & & & 1.00 & $0.97,1.03$ & 1.00 & $0.97,1.04$ \\
\hline \multicolumn{8}{|l|}{ Citrus fruit intake } \\
\hline Q1 & 42 & 5550 & $8 \cdot 2$ & 1 & & 1 & \\
\hline Q2 & 44 & 5858 & $38 \cdot 2$ & 0.99 & $0.64,1.55$ & 1.05 & $0 \cdot 66,1.66$ \\
\hline Q3 & 36 & 5618 & $77 \cdot 7$ & 0.85 & $0.53,1.35$ & $0 \cdot 87$ & $0.54,1.42$ \\
\hline Q4 & 31 & 5626 & $110 \cdot 9$ & 0.72 & $0.44,1.18$ & 0.79 & $0.47,1.31$ \\
\hline Q5 & 39 & 5383 & $187 \cdot 5$ & 0.96 & $0.60,1.52$ & 1.05 & $0.65,1.69$ \\
\hline$P$ for trend & & & & & 0.62 & & 0.89 \\
\hline Continuous ( $25 \mathrm{~g} / \mathrm{d}$ increment) & & & & 0.98 & $0.93,1.03$ & 0.98 & $0.93,1.04$ \\
\hline
\end{tabular}

Q, quintile.

${ }^{*}$ Adjusted for age (years); calculated using Cox proportional hazards model.

†Adjusted for age (years), smoking status (current $v$. non-current smoker), duration of cigarette smoking (years), number of cigarettes smoked per d, total energy intake (kJ/d), BMI (quintiles), alcohol intake ( $\mathrm{g} / \mathrm{d})$, levels of education (four categories), non-occupational physical activity (four categories) and use of lower oesophageal sphincter-relaxing medications (yes/no); calculated using Cox proportional hazards model.

vegetables, but significant inverse associations were not detected. HR estimates for cooked leafy vegetables suggested positive associations, but the estimate for the highest category was not significant. HR estimates were generally higher than those found among men, and interaction analyses with continuous variables suggested effect modification by sex for leafy vegetables (Wald: $P=0.04$ and $P=0.07$ for raw and cooked leafy vegetables, respectively), but not for other vegetable groups.

Table 4. Hazard ratios (HR) for Barrett's oesophagus with specialised intestinal metaplasia in men by nitrate intake from diet and water in the Netherlands Cohort Study on diet and cancer, 1986-2002

(Hazard ratios and $95 \%$ confidence intervals)

\begin{tabular}{|c|c|c|c|c|c|c|c|}
\hline & \multirow[b]{2}{*}{ Cases $(n)$} & \multicolumn{2}{|c|}{ Subcohort } & \multirow[b]{2}{*}{$\mathrm{HR}^{*}$} & \multirow[b]{2}{*}{$95 \% \mathrm{Cl}$} & \multirow[b]{2}{*}{$\mathrm{HR} \dagger$} & \multirow[b]{2}{*}{$95 \% \mathrm{Cl}$} \\
\hline & & Person-years & Median intake $(\mathrm{mg} / \mathrm{d})$ & & & & \\
\hline \multicolumn{8}{|l|}{ Nitrate intake from food } \\
\hline Q1 & 58 & 4724 & $58 \cdot 3$ & 1 & & 1 & \\
\hline Q2 & 47 & 5280 & $80 \cdot 0$ & 0.71 & $0.47,1.08$ & 0.72 & $0.47,1.11$ \\
\hline Q3 & 57 & 5258 & $99 \cdot 3$ & 0.88 & $0.59,1.31$ & 0.89 & $0.57,1.41$ \\
\hline Q4 & 45 & 5094 & $119 \cdot 8$ & 0.71 & $0.46,1.07$ & 0.72 & $0.42,1.23$ \\
\hline Q5 & 34 & 4842 & $157 \cdot 6$ & 0.56 & $0.36,0.88$ & 0.59 & $0.30,1 \cdot 18$ \\
\hline$P$ for trend & & & & & 0.02 & & $0 \cdot 19$ \\
\hline Continuous (10 mg/d increment) & & & & 0.96 & $0.92,1.00$ & 0.96 & $0.90,1.03$ \\
\hline \multicolumn{8}{|l|}{ Nitrate intake from water } \\
\hline Q1 & 34 & 4855 & 0.15 & 1 & & 1 & \\
\hline Q2 & 44 & 4967 & 0.50 & 1.28 & $0.80,2.05$ & $1 \cdot 30$ & $0.80,2.09$ \\
\hline Q3 & 48 & 5012 & $1 \cdot 16$ & 1.36 & $0.85,2.17$ & 1.38 & $0.86,2 \cdot 21$ \\
\hline Q4 & 52 & 5180 & 1.93 & 1.44 & $0.91,2.28$ & 1.48 & $0.93,2.35$ \\
\hline Q5 & 61 & 4707 & 3.60 & 1.83 & $1 \cdot 17,2 \cdot 87$ & 1.92 & $1.22,3.04$ \\
\hline$P$ for trend & & & & & 0.008 & & 0.005 \\
\hline Continuous ( $1 \mathrm{mg} / \mathrm{d}$ increment) & & & & 1.09 & $1.03,1.15$ & $1 \cdot 10$ & $1.04,1 \cdot 17$ \\
\hline \multicolumn{8}{|l|}{ Total nitrate intake $\ddagger$} \\
\hline Q1 & 59 & 4757 & 59.5 & 1 & & 1 & \\
\hline Q2 & 48 & 5250 & $81 \cdot 7$ & 0.73 & $0.48,1.10$ & 0.71 & $0.46,1 \cdot 10$ \\
\hline Q3 & 57 & 5298 & $100 \cdot 8$ & 0.86 & $0.58,1.28$ & 0.85 & $0.54,1.34$ \\
\hline Q4 & 45 & 5042 & 121.5 & 0.71 & $0.47,1.08$ & 0.68 & $0.40,1.15$ \\
\hline Q5 & 32 & 4852 & $159 \cdot 4$ & 0.52 & $0.33,0.82$ & 0.50 & $0.25,0.99$ \\
\hline$P$ for trend & & & & & 0.008 & & 0.07 \\
\hline Continuous ( $10 \mathrm{mg} / \mathrm{d}$ increment) & & & & 0.96 & $0.92,1.00$ & 0.97 & $0.91,1.04$ \\
\hline
\end{tabular}

Q, quintile.

${ }^{*}$ Adjusted for age (years); calculated using Cox proportional hazards model.

† Adjusted for age (years), smoking status (current $v$. non-current smoker), duration of cigarette smoking (years), number of cigarettes smoked per d, total energy intake (kJ/d), vegetable intake (g/d), fruit intake ( $\mathrm{g} / \mathrm{d})$, BMl (quintiles), alcohol intake ( $\mathrm{g} / \mathrm{d})$, levels of education (four categories), non-occupational physical activity (four categories) and use of lower oesophageal sphincter-relaxing medications (yes/no); calculated using Cox proportional hazards model.

$\ddagger$ Total nitrate is the sum of nitrate intake from food and nitrate intake from water. 
Table 5. Hazard ratios (HR) for Barrett's oesophagus with specialised intestinal metaplasia in women by nitrate intake from diet and water in the Netherlands Cohort Study on diet and cancer, 1986-2002

(Hazard ratios and $95 \%$ confidence intervals)

\begin{tabular}{|c|c|c|c|c|c|c|c|}
\hline & \multirow[b]{2}{*}{ Cases $(n)$} & \multicolumn{2}{|c|}{ Subcohort } & \multirow[b]{2}{*}{$\mathrm{HR}^{*}$} & \multirow[b]{2}{*}{$95 \% \mathrm{Cl}$} & \multirow[b]{2}{*}{$\mathrm{HR} \dagger$} & \multirow[b]{2}{*}{$95 \% \mathrm{Cl}$} \\
\hline & & Person-years & Median intake (mg/d) & & & & \\
\hline \multicolumn{8}{|l|}{ Nitrate intake from food } \\
\hline Q1 & 29 & 5468 & $57 \cdot 4$ & 1 & & 1 & \\
\hline Q2 & 39 & 5818 & $77 \cdot 3$ & 1.27 & $0.77,2.09$ & 1.51 & $0.88,2.60$ \\
\hline Q3 & 49 & 5928 & $97 \cdot 0$ & 1.56 & $0.96,2.52$ & $2 \cdot 26$ & $1.31,3.92$ \\
\hline Q4 & 39 & 5793 & $119 \cdot 4$ & $1 \cdot 28$ & $0.78,2 \cdot 12$ & $2 \cdot 12$ & $1.12,4.01$ \\
\hline $\begin{array}{l}\text { Q5 } \\
P \text { for trend }\end{array}$ & 36 & 5027 & $158 \cdot 3$ & $1 \cdot 35$ & $0.81,2.25$ & 3.25 & $\begin{array}{c}1.46,7.23 \\
0.005\end{array}$ \\
\hline Continuous ( $10 \mathrm{mg} / \mathrm{d}$ increment) & & & & 1.00 & $0.97,1.04$ & 1.07 & $1.00,1.15$ \\
\hline \multicolumn{8}{|l|}{ Nitrate intake from water } \\
\hline Q1 & 25 & 5473 & $0 \cdot 16$ & 1 & & 1 & \\
\hline Q2 & 42 & 5536 & 0.48 & 1.68 & $1 \cdot 00,2 \cdot 81$ & 1.84 & $1 \cdot 08,3 \cdot 13$ \\
\hline Q3 & 42 & 5305 & $1 \cdot 16$ & $1 \cdot 76$ & $1.05,2.94$ & $2 \cdot 01$ & $1 \cdot 18,3.41$ \\
\hline Q4 & 40 & 5718 & 1.97 & 1.54 & $0.92,2.60$ & $1 \cdot 71$ & $1.00,2.92$ \\
\hline Q5 & 38 & 5524 & 3.86 & 1.52 & $0.90,2.58$ & 1.67 & $0.97,2.89$ \\
\hline$P$ for trend & & & & & 0.52 & & 0.39 \\
\hline Continuous ( $1 \mathrm{mg} / \mathrm{d}$ increment) & & & & 1.01 & $0.95,1.07$ & 1.01 & $0.96,1.07$ \\
\hline \multicolumn{8}{|l|}{ Total nitrate intake $\neq$} \\
\hline Q1 & 28 & 5534 & $58 \cdot 6$ & 1 & & 1 & \\
\hline Q2 & 44 & 5721 & $79 \cdot 3$ & 1.53 & $0.93,2.51$ & 1.84 & $1.07,3.15$ \\
\hline Q3 & 45 & 5980 & 98.5 & 1.49 & $0.91,2.44$ & $2 \cdot 15$ & $1.22,3.79$ \\
\hline Q4 & 37 & 5764 & 121.7 & $1 \cdot 28$ & $0.77,2 \cdot 15$ & $2 \cdot 19$ & $1 \cdot 15,4 \cdot 18$ \\
\hline Q5 & 38 & 5035 & 159.5 & 1.50 & $0.90,2.49$ & 3.77 & $1.68,8.45$ \\
\hline$P$ for trend & & & & & 0.33 & & 0.004 \\
\hline Continuous (10 mg/d increment) & & & & 1.00 & $0.97,1.04$ & 1.07 & $1.00,1.15$ \\
\hline
\end{tabular}

Q, quintile.

${ }^{*}$ Adjusted for age (years); calculated using Cox proportional hazards model.

† Adjusted for age (years), smoking status (current $v$. non-current smoker), duration of cigarette smoking (years), number of cigarettes smoked per d, total energy intake $(\mathrm{kJ} / \mathrm{d})$, vegetable intake $(\mathrm{g} / \mathrm{d})$, fruit intake $(\mathrm{g} / \mathrm{d})$, BMI (quintiles), alcohol intake $(\mathrm{g} / \mathrm{d})$, levels of education (four categories), non-occupational physical activity (four categories) and use of lower oesophageal sphincter-relaxing medications (yes/no); calculated using Cox proportional hazards model.

$\ddagger$ Total nitrate is the sum of nitrate intake from food and nitrate intake from water.

Associations between nitrate intake and Barrett's oesophagus risk in men and women are summarised in Tables 4 and 5, respectively. The highest $v$. the lowest category of total nitrate and nitrate intake from food was inversely associated with Barrett's oesophagus risk among men after adjustment for confounders including vegetables. However, the estimate for nitrate intake from food in the multivariable model was not significant. Nitrate intake from water was positively associated with Barrett's oesophagus risk. Among women, estimates for the highest category suggested moderately strong positive associations for all the three nitrate variables. Test for interaction with sex was significant for age-adjusted and multivariable models with categorical exposure variables for total nitrate intake $(P=0.04)$. Interaction between nitrate intake and vitamin $\mathrm{C}$ intake was not significant ( $P=0.10$ and $P=0.99$ in men and women, respectively).

Analysis restricted to individuals who reported having similar vegetable and fruit intakes 5 years earlier was based on 104 male and 89 female cases and 1632 subcohort members. An association with total vegetable intake was no longer apparent among men (HR for the highest $v$. the lowest category: $0 \cdot 81,95 \%$ CI $0 \cdot 40,1 \cdot 65$ ). The HR for the highest category of raw vegetable intake compared with the lowest category was $0.38(95 \% \mathrm{CI} 0 \cdot 18,0.80)$ in men and $0.94(95 \%$ CI $0.45,1.95)$ in women $(P$ for interaction $=0.79)$. Analyses for nitrate intake were similar to the primary analysis with somewhat attenuated HR estimates (data not shown). Analyses excluding the first 2 years of follow-up did not substantially change the estimates for vegetable/fruit intake and nitrate intake (data not shown).

\section{Discussion}

This is the first prospective cohort study to examine the association between vegetable and fruit consumption, as well as nitrate intake, and Barrett's oesophagus risk. The results indicate an inverse association between total vegetable intake and Barrett's oesophagus risk among men. Raw vegetable intake, especially raw leafy vegetable intake, was inversely associated with Barrett's oesophagus risk in men and the highest category of Brassica vegetable intake also suggested a protective effect. Estimates among women, in general, do not suggest an inverse association. Fruit consumption was not associated with Barrett's disease risk in men or in women. In men, an inverse association with nitrate intake was observed, but not with nitrate intake from water sources. In women, nitrate intake was positively associated with Barrett's disease risk. Effects were not different across the levels of vitamin $\mathrm{C}$ intake.

Epidemiological studies have suggested an inverse association between vegetable and fruit intake and oesophageal adenocarcinoma, and stronger inverse associations have been reported with the intake of green leafy, raw and 
cruciferous vegetables in several studies ${ }^{(25)}$. Few studies have examined the association between vegetable and fruit intake and Barrett's disease, a precursor lesion for adenocarcinoma. Case-control studies have observed an inverse association with vegetable and/or fruit intake ${ }^{(26-29)}$, a 'health-conscious' diet including fruits and vegetables ${ }^{(30)}$, and fibre intake ${ }^{(31,32)}$. A case-control study carried out in the USA has evaluated specific subtypes of vegetables and found an inverse association with dark green vegetables ${ }^{(33)}$. The present results suggest that a possible protective effect could be due to raw leafy vegetable and cruciferous vegetable consumption, in line with previous findings for oesophageal adenocarcinoma $^{(25)}$ and Barrett's oesophagus ${ }^{(33)}$.

Vegetables are a major source of nitrate in human diet. Nitrate intake might be responsible for some of the effects of raw leafy vegetables found in the present study. Absorbed nitrate is excreted by the salivary glands in high concentrations and is reduced to nitrite by anaerobic bacteria ${ }^{(9)}$. High concentrations of NO are generated from nitrite at the gastro-oesophageal junction under acidic conditions ${ }^{(34)}$, and high concentrations of NO can also be found within the columnar lined oesophagus of patients with Barrett's oesophagus during acid reflux ${ }^{(35)}$. It has thus been suggested that high doses of NO in the oesophagus may be important in the development of Barrett's oesophagus ${ }^{(36)}$, possibly through its effect on oesophageal tissue damage ${ }^{(37)}$. It has also been shown that dietary nitrate may induce $N$-nitrosation in juxtaluminal compartments of the upper gastrointestinal tract of healthy subjects and patients with Barrett's oesophagus via the generation of nitric oxide ${ }^{(38)}$. In the absence of vitamin $\mathrm{C}$ and under acidic conditions, nitrite is converted to nitrous acid and nitrosating agents that can react with secondary amines to form $N$-nitrosamines ${ }^{(39)}$. On the other hand, ingested nitrate also has several beneficial effects through the formation of $\mathrm{NO}^{(40)}$, including protection of gastric mucosa from damage $^{(41,42)}$ and from gastrointestinal infections ${ }^{(43,44)}$. We are not aware of any previous epidemiological studies that have evaluated the association between nitrate intake and Barrett's oesophagus risk. Studies assessing the risk of oesophageal adenocarcinoma did not show associations with nitrate intake from diet and/or drinking water ${ }^{(11-13)}$. Although the present results cannot be unambiguously explained by the possible positive and negative effects of nitrate in the gastro-oesophageal junction, our findings provide additional support to the hypothesis that increased nitrate intake may increase or decrease the risk of health outcomes in different populations ${ }^{(40)}$.

Previous epidemiological studies did not evaluate the possible differences between men and women regarding the association of Barrett's disease risk with vegetable intake ${ }^{(26-28)}$. The present results suggest a differential effect of raw vegetables in men and women, which might be partly explained by the sex differences in the occurrence of Barrett's oesophagus and differences in oesophageal pathophysiology. Barrett's disease occurs more often in men with a male to female ratio of 2:1 estimated in a meta-analysis ${ }^{(45)}$, and male sex is an independent risk factor for oesophagitis ${ }^{(46,47)}$. Studies comparing men and women with reflux symptoms suggest sexspecific differences in oesophageal acid exposure, presence of defective oesophageal sphincter and hiatus hernia ${ }^{(48)}$. Animal studies have suggested that female sex hormones might have an effect on parietal cell mass and decrease basal acid secretion $^{(49)}$, and in rat models of reflux oesophagitis, oesophageal damage has been shown to be more prominent in male rats than in female rats in the presence of $\mathrm{NO}$ administration ${ }^{(50)}$. These sex-specific differences support the hypothesis that dietary factors might have differential effects on the progression from reflux disease to Barrett's oesophagus. However, if these experimental results translate to effects on human risks, they would suggest more protective effects of sex hormones in women rather than in men.

Differential bias could also partly explain our dissimilar findings in men and women. Since the absence of Barrett's disease in the cohort of the present study cannot be verified, false-negative cases might have occurred. A previous study investigating the presence of Barrett's oesophagus among asymptomatic individuals has found a prevalence of $3 \%$ in men and $1 \%$ among women ${ }^{(51)}$. We cannot rule out the possibility that different false-negative proportions among men and women could have led to differential bias in the present study.

Other limitations include the lack of information on gastrooesophageal reflux and Helicobacter pylori infection, which has been shown to be inversely associated with Barrett's oesophagus ${ }^{(52)}$. Additionally, it is difficult to measure vegetable and fruit intake in large epidemiological studies. Low correlations were observed for vegetable intake in the validation of the FFQ ${ }^{(17)}$. We would expect to find attenuations of $\mathrm{HR}$ estimates, and it is unlikely that differential bias across cases and non-cases would have been introduced. Similarly, attenuation of estimates might have resulted from the lack of repeated exposure measurements over time.

The prospective design, the large number of cases and the availability of full pathology reports to identify intestinal metaplasia are the most important strengths of the present study.

In conclusion, results obtained for this large prospective cohort are consistent with an inverse association between vegetable intake, especially green leafy vegetable intake, and Barrett's oesophagus risk. These findings add to the limited number of published epidemiological research on the relationship between vegetable intake and Barrett's oesophagus risk. However, the possibility that the beneficial effects of vegetables might be stronger among men should be evaluated further.

\section{Acknowledgements}

The authors thank PALGA and the pathologist for supplying the pathology reports of Barrett's disease cases. They also thank Dr A. Volovics and Dr A. Kester for their statistical advice; S. van de Crommert, H. Brants, J. Nelissen, C. de Zwart, M. Moll, W. van Dijk, M. Jansen, Ellen Dutman and A. Pisters for their assistance; and $\mathrm{H}$. van Montfort, T. van Moergastel, L. van den Bosch and R. Schmeitz for their programming assistance.

The present study was supported by a grant from Maag Lever Darm Stichting (MLDS) (WO 09-53). MLDS had no role 
in the design and analysis of the study or in the writing of this article.

The authors' contributions are as follows: R. A. G., L. J. S., Y. C. A. K. and P. A. vd B. designed the research; R. A. G., L. J. S. and P. A. vd B. were involved in the coordination of the study; A. P. K., A. L. C. D., C. J. R. H. and L. J. S. conducted the research; A. P. K. analysed the data and wrote the article; P. A. vd B. had primary responsibility for the final content. All authors read and approved the final manuscript.

None of the authors has a conflict of interest to declare.

\section{References}

1. Botterweck AA, Schouten LJ, Volovics A, et al. (2000) Trends in incidence of adenocarcinoma of the oesophagus and gastric cardia in ten European countries. Int $J$ Epidemiol 29, 645-654

2. van Blankenstein M, Looman CW, Siersema PD, et al. (2007) Trends in the incidence of adenocarcinoma of the oesophagus and cardia in the Netherlands 1989-2003. Br J Cancer 96, $1767-1771$.

3. Trivers KF, Sabatino SA \& Stewart SL (2008) Trends in esophageal cancer incidence by histology, United States, 1998-2003. Int J Cancer 123, 1422-1428.

4. Steevens J, Botterweck AA, Dirx MJ, et al. (2010) Trends in incidence of oesophageal and stomach cancer subtypes in Europe. Eur J Gastroenterol Hepatol 22, 669-678.

5. Coleman HG, Bhat $\mathrm{S}$, Murray LJ, et al. (2011) Increasing incidence of Barrett's oesophagus: a population-based study. Eur J Epidemiol 26, 739-745.

6. Ruol A, Parenti A, Zaninotto G, et al. (2000) Intestinal metaplasia is the probable common precursor of adenocarcinoma in Barrett's esophagus and adenocarcinoma of the gastric cardia. Cancer 88, 2520-2528.

7. Winberg H, Lindblad M, Lagergren J, et al. (2012) Risk factors and chemoprevention in Barrett's esophagus - an update. Scand J Gastroenterol 47, 397-406.

8. De Ceglie A, Fisher DA, Filiberti R, et al. (2011) Barrett's esophagus, esophageal and esophagogastric junction adenocarcinomas: the role of diet. Clin Res Hepatol Gastroenterol $35,7-16$.

9. Lundberg JO, Weitzberg E, Cole JA, et al. (2004) Nitrate, bacteria and human health. Nat Rev Microbiol 2, 593-602.

10. Tricker AR (1997) $\mathrm{N}$-nitroso compounds and man: sources of exposure, endogenous formation and occurrence in body fluids. Eur J Cancer Prev 6, 226-268.

11. Cross AJ, Freedman ND, Ren J, et al. (2011) Meat consumption and risk of esophageal and gastric cancer in a large prospective study. Am J Gastroenterol 106, 432-442.

12. Keszei AP, Goldbohm RA, Schouten LJ, et al. (2013) Dietary $\mathrm{N}$-nitroso compounds, endogenous nitrosation, and the risk of esophageal and gastric cancer subtypes in the Netherlands Cohort Study. Am J Clin Nutr 97, 135-146.

13. Ward MH, Heineman EF, Markin RS, et al. (2008) Adenocarcinoma of the stomach and esophagus and drinking water and dietary sources of nitrate and nitrite. Int J Occup Environ Health 14, 193-197.

14. van den Brandt PA, Goldbohm RA, van 't Veer P, et al. (1990) A large-scale prospective cohort study on diet and cancer in The Netherlands. J Clin Epidemiol 43, 285-295.

15. Steevens J, Schouten LJ, Driessen AL, et al. (2010) A prospective cohort study on overweight, smoking, alcohol consumption, and risk of Barrett's esophagus. Cancer Epidemiol Biomarkers Prev 20, 345-358.
16. Steevens J, Schouten LJ, Goldbohm RA, et al. (2011) Vegetables and fruits consumption and risk of esophageal and gastric cancer subtypes in the Netherlands Cohort Study. Int J Cancer 129, 2681-2693.

17. Goldbohm RA, van den Brandt PA, Brants HA, et al. (1994) Validation of a dietary questionnaire used in a large-scale prospective cohort study on diet and cancer. Eur J Clin Nutr 48, 253-265.

18. van de Worp HHM (1987) Onderzoek naar de gehalte aan nitraat en nitriet in een aantal wintergroenten. (Examination of the Nitrate and Nitrite Content of Various Winter Vegetables). Wageningen: State Institute for Quality Control of Agricultural Products (RIKILT).

19. Driessen JJM (1989) Nitraat-en nitrietgehalte van een aantal rauwe en toebereide groenten. (Nitrate and Nitrite Content of a Number of Raw and Cooked Vegetables). Wageningen: State Institute for Quality Control of Agricultural Products (RIKILT)

20. van Loon AJM \& van Klaveren JD (1991) Nitraatinname van de Nederlandse bevolking (Nitrate intake of the Dutch population). Voeding 52, 96-100.

21. Barlow WE, Ichikawa L, Rosner D, et al. (1999) Analysis of case-cohort designs. J Clin Epidemiol 52, 1165-1172.

22. Lin DY \& Wei LJ (1989) The robust inference for the Cox proportional hazards model. J Am Stat Assoc 84, 1074-1078.

23. Schoenfeld D (1982) Partial residuals for the proportional hazards regression model. Biometrika 69, 239-241.

24. Cai J \& Zeng D (2004) Sample size/power calculation for case-cohort studies. Biometrics 60, 1015-1024.

25. Kubo A, Corley DA, Jensen CD, et al. (2010) Dietary factors and the risks of oesophageal adenocarcinoma and Barrett's oesophagus. Nutr Res Rev 23, 230-246.

26. Anderson LA, Watson RG, Murphy SJ, et al. (2007) Risk factors for Barrett's oesophagus and oesophageal adenocarcinoma: results from the FINBAR study. World $J$ Gastroenterol 13, 1585-1594.

27. Kubo A, Levin TR, Block G, et al. (2008) Dietary antioxidants, fruits, and vegetables and the risk of Barrett's esophagus. Am J Gastroenterol 103, 1614-1623.

28. Thompson OM, Beresford SA, Kirk EA, et al. (2009) Vegetable and fruit intakes and risk of Barrett's esophagus in men and women. Am J Clin Nutr 89, 890-896.

29. Pohl H, Wrobel K, Bojarski C, et al. (2013) Risk factors in the development of esophageal adenocarcinoma. Am J Gastroenterol 108, 200-207.

30. Kubo A, Levin TR, Block G, et al. (2008) Dietary patterns and the risk of Barrett's esophagus. Am J Epidemiol 167, 839-846

31. Kubo A, Block G, Quesenberry CP, et al. (2009) Effects of dietary fiber, fats, and meat intakes on the risk of Barrett's esophagus. Nutr Cancer 61, 607-616.

32. Mulholland HG, Cantwell MM, Anderson LA, et al. (2009) Glycemic index, carbohydrate and fiber intakes and risk of reflux esophagitis, Barrett's esophagus, and esophageal adenocarcinoma. Cancer Causes Control 20, 279-288.

33. Jiao L, Kramer JR, Rugge M, et al. (2013) Dietary intake of vegetables, folate, and antioxidants and the risk of Barrett's esophagus. Cancer Causes Control 24, 1005-1014.

34. Iijima K, Henry E, Moriya A, et al. (2002) Dietary nitrate generates potentially mutagenic concentrations of nitric oxide at the gastroesophageal junction. Gastroenterology 122, 1248-1257.

35. Suzuki H, Iijima K, Scobie G, et al. (2005) Nitrate and nitrosative chemistry within Barrett's oesophagus during acid reflux. Gut 54, 1527-1535. 
36. Iijima K \& Shimosegawa T (2006) Gastric carditis: is it a histological response to high concentrations of luminal nitric oxide? World J Gastroenterol 12, 5767-5771.

37. Ishiyama F, Iijima K, Asanuma K, et al. (2009) Exogenous luminal nitric oxide exacerbates esophagus tissue damage in a reflux esophagitis model of rats. Scand J Gastroenterol 44, 527-537.

38. Winter JW, Paterson S, Scobie G, et al. (2007) $N$-nitrosamine generation from ingested nitrate via nitric oxide in subjects with and without gastroesophageal reflux. Gastroenterology 133, 164-174.

39. Mirvish SS (1995) Role of $N$-nitroso compounds (NOC) and $\mathrm{N}$-nitrosation in etiology of gastric, esophageal, nasopharyngeal and bladder cancer and contribution to cancer of known exposures to NOC. Cancer Lett 93, 17-48.

40. Gilchrist M, Winyard PG \& Benjamin N (2010) Dietary nitrate - good or bad? Nitric Oxide 22, 104-109.

41. Kitagawa H, Takeda F \& Kohei H (1990) Effect of endothelium-derived relaxing factor on the gastric lesion induced by $\mathrm{HCl}$ in rats. J Pharmacol Exp Ther 253, 1133-1137.

42. Miyoshi M, Kasahara E, Park AM, et al. (2003) Dietary nitrate inhibits stress-induced gastric mucosal injury in the rat. Free Radic Res 37, 85-90.

43. Dykhuizen RS, Frazer R, Duncan C, et al. (1996) Antimicrobial effect of acidified nitrite on gut pathogens: importance of dietary nitrate in host defense. Antimicrob Agents Chemother 40, 1422-1425.
44. Dykhuizen RS, Fraser A, McKenzie H, et al. (1998) Helicobacter pylori is killed by nitrite under acidic conditions. Gut 42, 334-337.

45. Cook MB, Wild CP \& Forman D (2005) A systematic review and meta-analysis of the sex ratio for Barrett's esophagus, erosive reflux disease, and nonerosive reflux disease. $A m \mathrm{~J}$ Epidemiol 162, 1050-1061

46. El-Serag HB \& Johanson JF (2002) Risk factors for the severity of erosive esophagitis in Helicobacter pylori-negative patients with gastroesophageal reflux disease. Scand J Gastroenterol 37, 899-904.

47. Ford AC, Forman D, Reynolds PD, et al. (2005) Ethnicity, gender, and socioeconomic status as risk factors for esophagitis and Barrett's esophagus. Am J Epidemiol 162, 454-460.

48. Banki F, Demeester SR, Mason RJ, et al. (2005) Barrett's esophagus in females: a comparative analysis of risk factors in females and males. Am J Gastroenterol 100, 560-567.

49. Adeniyi KO (1991) Gastric acid secretion and parietal cell mass: effect of sex hormones. Gastroenterology 101, 66-69.

50. Masaka T, Iijima K, Endo H, et al. (2013) Gender differences in oesophageal mucosal injury in a reflux oesophagitis model of rats. Gut 62, 6-14.

51. Fan X \& Snyder N (2009) Prevalence of Barrett's esophagus in patients with or without GERD symptoms: role of race, age, and gender. Dig Dis Sci 54, 572-577.

52. Thrift AP, Pandeya N, Smith KJ, et al. (2012) Helicobacter pylori infection and the risks of Barrett's oesophagus: a population-based case-control study. Int J Cancer 130, $2407-2416$. 